\title{
Insectos acuáticos asociados a Eichhornia azurea (Schwartz) Kunth en ciénagas del río Atrato, Chocó - Colombia
}

\author{
Aquatic insects associated to Eichhornia azurea (Schwartz) Kunth in wetlands \\ of Atrato River, Chocó - Colombia
}

\author{
Mosquera-Murillo, Zuleymaํㅗ.Sc. \\ ¡Universidad Tecnológica del Chocó, Facultad de Ciencias Naturales, Grupo de Limnología, Quibdó-Chocó.
}

\section{KEYWORDS:}

Entomofauna aquatic; Eichhornia azurea; floodplain;

Atrato river.

\section{PALABRAS CLAVE:}

INFORMACIÓN

Recibido: 25-07-2017;

Aceptado: 24-11-2017.

Correspondencia autor:

d-zuleyma.mosquera@utch.edu.co

\begin{abstract}
The objective of this study was to characterize the aquatic insect community associated with Eichhornia azurea (Schwartz) Kunth in four swamp of the middle and lower basin of the Atrato river (Chocó-Colombia) during high and low water periods; the influence of environmental and physicochemical variables on this community was also evaluated. For two months, aquatic insects associated with the roots of $E$. azurea were collected, using a quadrant of one $\mathrm{m}^{2}$, provided with a mesh of $0.5 \mathrm{~mm}$, with three replicates per swamp and simultaneously measured some physical and chemical variables of the water. The community consisted of 6 orders, 21 families and 28 genera. The highest number of organisms $(55.39 \%)$ and of taxa (23) were recorded in the low water period. The most abundant orders were Ephemeroptera (37.55\%) and Coleoptera (26.77\%), standing out the families Baetidae and Noteridae respectively; while the greatest taxonomic richness was for Coleoptera, Diptera and Hemiptera. There were no significant differences in diversity and dominance between hydrological periods ( $p>0.05)$, but in the specific richness $(p<0.05)$. The most abundant functional groups were predators $(54.81 \%)$ and collectors $(43.70 \%)$. The physicochemical variables did not show significant differences between hydrological periods and there were associations between the aquatic entomofauna and the abiotic variables analyzed, mainly with dissolved oxygen, conductivity and total dissolved solids.
\end{abstract}

\section{RESUMEN}

El objetivo de este estudio fue caracterizar la comunidad de insectos acuáticos asociados a Eichhornia azurea (Schwartz) Kunth en cuatro ciénagas de la cuenca media y baja del río Atrato (Chocó-Colombia) durante los periodos de aguas altas y bajas; igualmente se evaluó la influencia de las variables ambientales y fisicoquímicas sobre esta comunidad. Durante dos meses se hicieron colectas de insectos acuáticos asociados a las raíces de $E$. azurea, utilizando un cuadrante de un $\mathrm{m}^{2}$, dotado de una malla de $0,5 \mathrm{~mm}$, con tres replicas por ciénaga y simultáneamente se midieron algunas variables físicas y químicas del agua. La comunidad estuvo compuesta por 6 órdenes, 21 familias y 28 géneros. El mayor número de organismos (55,39\%) y de taxones (23) se registró en el periodo de aguas bajas. Los órdenes más abundantes fueron Ephemeroptera (37,55\%) y Coleoptera $(26,77 \%)$, sobresaliendo las familias Baetidae y Noteridae respectivamente; mientras que la mayor riqueza taxonómica fue para coleópteros, dípteros y hemípteros. No se presentaron diferencias significativas en la diversidad y dominancia entre periodos hidrológicos $(p>0,05)$, pero si en la riqueza específica $(p<0,05)$. Los grupos funcionales de mayor abundancia fueron predadores $(54,81 \%)$ y colectores $(43,70 \%)$. Las variables fisicoquímicas hídricas no mostraron diferencias significativas entre periodos hidrológicos y se presentaron asociaciones entre la entomofauna acuática y las variables abióticas analizadas, principalmente con el oxígeno disuelto, la conductividad y los sólidos totales disueltos. 


\section{INTRODUCCIÓN}

Las ciénagas o lagos de planos inundables de zonas bajas, son cuerpos de agua poco profundos con conexión directa y/o indirecta a un río de forma temporal o permanente; tienen una columna de agua que no supera los $10 \mathrm{~m}$, presentan estratificación durante el día y mezcla en la noche (RíOS et al., 2008). En éstos, las plantas acuáticas o macrófitas juegan un papel fundamental, ya que son uno de los principales elementos en delinear la estructura física del hábitat, confieren mayor heterogeneidad y modulan los diferentes procesos ecológicos que tienen lugar en ellos; además, tienen una fuerte influencia en la abundancia, diversidad y en los atributos de las comunidades de los organismos acuáticos (FONTANARROSA et al., 2012).

Las macrófitas acuáticas tienen importancia ecológica reconocida por componer un hábitat particular para numerosos macroinvertebrados acuáticos, entre ellos insectos acuáticos que, a su vez, constituyen uno de los principales productores de biomasa en ambientes lénticos, haciendo los sitios donde ellos ocurren significativamente más productivos (STRIPARI y HENRY, 2002; MORETTI et al., 2003; MORMUL et al., 2006). Esta flora juega un papel ecológico importante en la estructuración de estas comunidades como fuente indirecta de alimentos para raspadores que utilizan la biopelícula que se adhiere a las paredes de las plantas $y$, directa para las especies minadoras y fragmentadoras, utilizando el tejido de la planta como fuente de alimento; además son un lugar de oviposición y emergencia de varios insectos acuáticos y actúan como filtros manteniendo las partículas orgánicas que pueden ser utilizadas por organismos detritívoros (TRIVINHO-STRIXINO et al., 2000; VILLANUEVA y TROCHINE, 2005; WALKER et al., 2013).

Eichhornia azurea (Swartz) Kunth (1843) es una de las macrófitas acuáticas de hojas flotantes, que presentan amplia distribución en ecosistemas de agua dulce de América (POTT y POTT, 2000). Sus raíces insertadas en el tallo se asemejan a largas cabelleras que se componen de múltiples ramificaciones. La longitud de sus raíces puede presentar grandes variaciones, pudiendo presentar unos pocos centímetros $(5 \mathrm{~cm})$ al inicio de su desarrollo, hasta tener un metro en las plantas más viejas (PADIAL et al., 2009). Esta especie de macrófitos en general, favorece la presencia de una rica y abundante macrofauna, en vista de que sus hojas sirven como fuente de alimento para las especies trituradoras cuando se encuentra en fase de senescencia y para especies colectoras que obtiene los recursos alimenticios a través de la acumulación de detritus en sus raíces (TRIVINHO-STRIXINO et al., 2007; MARTINS et al., 2011).
En el departamento del Chocó, estudios que ofrezcan conocimientos sobre las comunidades insectos acuáticos presentes en sistemas cenagosos son escasos, sobresaliendo los realizados por ASPRILLA et al. (1998) en la ciénaga de Jotaudó (Quibdó-Chocó); ÁLVAREZ y VIVAS (1999) en raíces de Pontederia rotundifolia (Algamasa) de la zona litoral y limnética en las ciénagas Plaza seca y La grande (SancenoQuibdó); ÁLVAREZ et al., (2003) compararon el bentos y los organismos asociados a macrófitas en un ambiente acuático temporal (Quibdó-Chocó) y más recientemente MOSQUERA-MURILLO y CÓRDOBA (2015), caracterizaron la comunidad de macroinvertebrados acuáticos en tres ciénagas de la cuenca media del Atrato, Chocó; por lo que no existe una línea base importante sobre las comunidades de insectos acuáticos asociadas a plantas acuáticas en sistemas cenagosos de la región. En general, en Colombia se destacan las investigaciones realizadas por MANJARREZ-HERNÁNDEZ (2004), INGER et al. (2004), OVALLE (2006), DELUQUE et al. (2006), VENEGAS (2008), MARTÍNEZ (2009), QUIROZ et al. (2010), RIVERA-USME (2011), RIVERA et al. (2013) entre otras.

El presente estudio tuvo como objetivo caracterizar la comunidad de insectos acuáticos asociados a las raíces de E. azurea, así como evaluar su relación con las condiciones abióticas en ciénagas de la cuenca media y baja del río Atrato, Chocó.

\section{MATERIALES Y MÉTODOS}

Área de estudio. La presente investigación se desarrolló en cuatro ciénagas ubicadas en la cuenca media y baja del río Atrato, Chocó (Figura 1), caracterizadas por presentar grandes parches de E. azurea. Esta zona presenta una precipitación promedio anual de 12000 $\mathrm{mm}$, temperatura promedio de $26,8^{\circ} \mathrm{C}$ y una humedad relativa del $85,7 \%$, correspondiendo a una zona de vida de bosque muy húmedo tropical (bmh-T) (RANGEL, 2004). Según DUQUE (1990), los sistemas cenagosos de esta región tienen un desarrollo genético de suelos muy incipientes, de la edad terciaria, arcillosos y permanecen inundados casi todo el año, localizados en las áreas más bajas (pendientes entre 1 y $8 \%$ ) poseen baja fertilidad, son químicamente ácidos, orgánicos, de textura generalmente fina, afectados por un acentuado intemperismo, color pardo o grisáceo oscuro, superficiales, pobres en nitrógeno, fósforo y potasio, con niveles intermedios de aluminio intercambiable (IGAC, 2006). 


\section{REcina Revista coldmbiana}

Mosquera-Murillo, Zuleyma
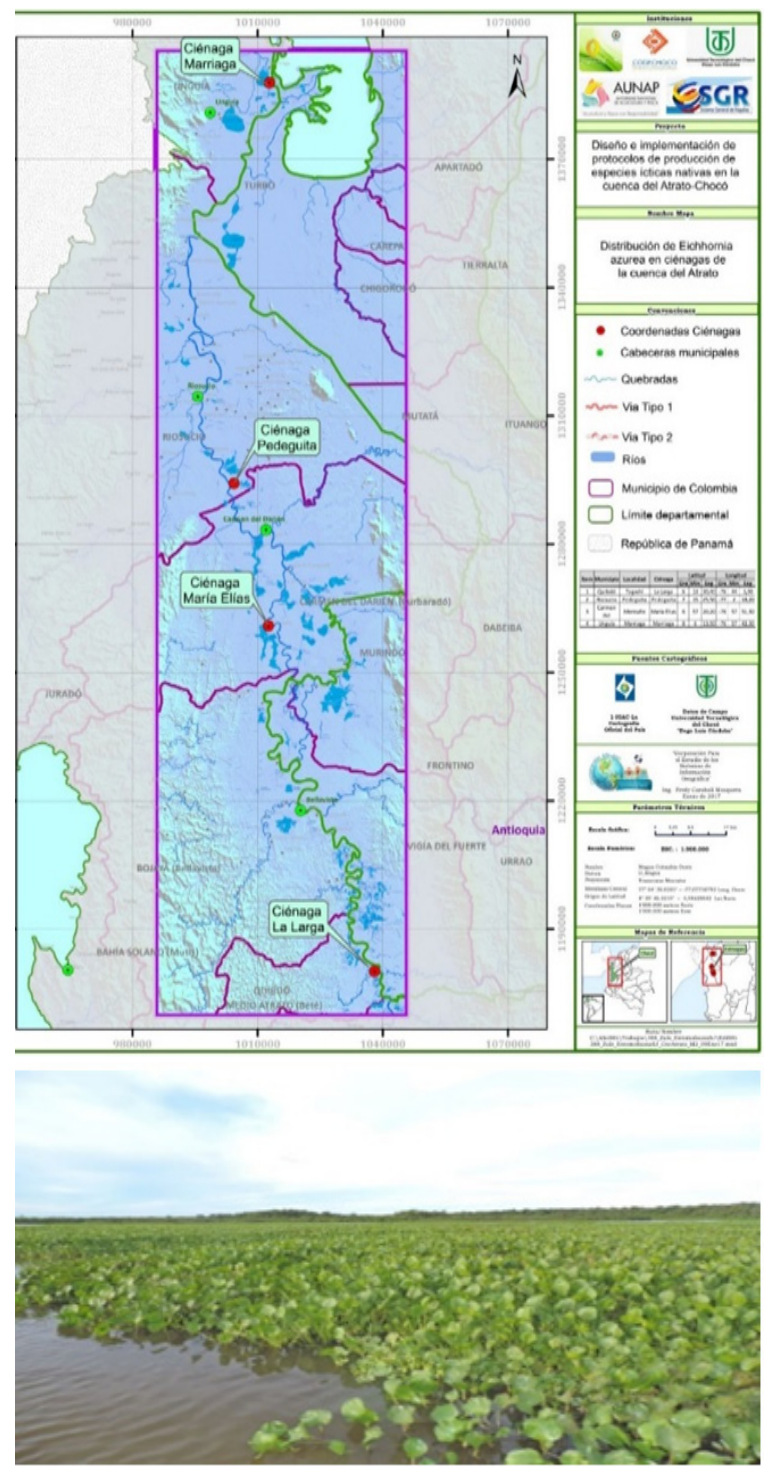

Figura 1. Área de estudio y presencia de E. azurea en una de las ciénagas estudiada, en la cuenca media y baja del río Atrato.

Trabajo de campo. La toma de datos se realizó entre los meses de agosto de 2014 y enero de 2015, abarcando los dos periodos hidrológicos de la región (aguas altas y aguas bajas) y en cada ciénaga estudiada se ubicó una estación. Para el muestreo de la comunidad de insectos acuáticos asociados a las raíces de $E$. azurea se utilizó un cuadrante de un $\mathrm{m}^{2}$, dotado de una malla de $0,5 \mathrm{~mm}$, con tres replicas por ciénaga. Éste se introdujo lenta y cuidadosamente en posición vertical, hasta alcanzar la longitud máxima de las raíces de las plantas, las cuales fueron extraídas y lavadas cuidadosamente para remover los organismos allí presentes y los insectos acuáticos colectados se preservaron en alcohol etílico al 90\%. De forma simultánea, se hicieron mediciones de algunas variables abióticas (fisicoquímicas e hidrológicas) con ayuda de un equipo digital multiparámetro Ysi. Los conteos y determinación taxonómica se realizaron en el laboratorio de Limnología de la Universidad Tecnológica del Chocó, con un estereomicroscopio NIKON SMZ 745 y las claves taxonómicas de DOMíNGUEZ et al. (2006), MERRIT et al. (2008), DOMÍNGUEZ y FERNÁNDEZ (2009) y cada taxón fue asignado a un grupo funcional de acuerdo a la clasificación propuesta por diversos autores (RIVERAUSME et al., 2013; CHARA et al., 2010).

Análisis de datos. La comunidad de insectos acuáticos asociada a $E$. azurea se caracterizó de acuerdo a las siguientes variables: número total de individuos, abundancia relativa de taxones y la clasificación de los grupos funcionales alimenticios. La estructura de la comunidad fue evaluada mediante los índices de diversidad de Shannon-Weaver, dominancia de Simpson y riqueza específica y sus diferencias entre periodos hidrológicos se evaluó mediante un ANOVA. Se realizó una prueba de Kruskal-Wallis para evaluar las condiciones abióticas de las ciénagas entre periodos hidrológicos y se utilizó el coeficiente de correlación de Pearson para relacionar la abundancia de insectos acuáticos asociados a $E$. azurea y las variables abióticas de los ecosistemas. Para estos análisis se emplearon los programas Minitab versión 17.1.0 (2014), Statgraphics Centurion XV (2006) y Past 1.57 (HAMMER et al., 2001).

\section{RESULTADOS}

Composición y estructura por grupos funcionales de la comunidad de insectos acuáticos asociados a $\boldsymbol{E}$. azurea. La comunidad de insectos acuáticos asociada a $E$. azurea estuvo compuesta por 28 géneros, distribuidos en 6 órdenes y 21 familias, los cuales son reportados por primera vez para las ciénagas estudiadas (Tabla 1). El mayor número de organismos se colectó en el periodo de aguas bajas con el $55,39 \%$ de los individuos, así como de taxones (23). De manera general, Ephemeroptera fue el orden más abundante con el $37,55 \%$ de los individuos, seguido de Coleoptera con el $26,77 \%$; mientras que los trichopteros presentaron la más baja abundancia con un 1,49\% (Figura 2). De las 21 familias reportadas, solo 3: Baetidae $(37,17 \%)$, Noteridae $(23,05 \%)$ y Chironomidae $(11,90 \%)$ se destacaron por presentar la mayor abundancia (Figura 3). La mayor riqueza genérica la registran los órdenes Coleoptera, Diptera y Hemiptera con 6 géneros cada uno (Figura 2).

La mayor diversidad de la entomofauna acuática se presenta en el periodo de aguas bajas $(1,59 \pm 1,27)$ y 
la menor en aguas altas $(1,25 \pm 0,42)$; igual situación ocurre para la riqueza con un mayor valor en aguas bajas (13) y el menor en aguas altas (5). Para la dominancia, por el contrario, se observó el mayor valor en aguas altas $(0,61 \pm 0,17)$ y el menor en aguas bajas $(0,57 \pm 0,45)$. El análisis de varianza muestra que no se presentaron diferencias significativas en la diversidad $(\mathrm{F}=0,30 ; p=0,614)$ y dominancia $(\mathrm{F}=0,04 ; p=0,853)$ entre periodos hidrológicos, pero si en la riqueza específica $(\mathrm{F}=15,61 ; p=0,017)$.

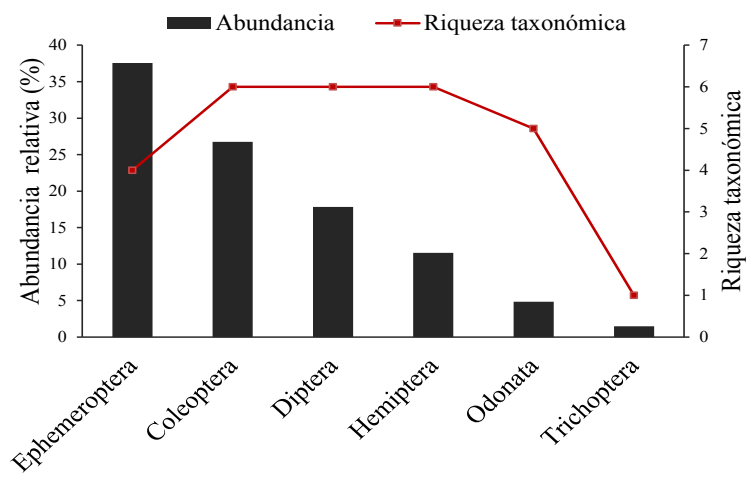

Figura 2. Abundancia relativa y riqueza taxonómica por órdenes de insectos acuáticos asociados a $E$. azurea.
La composición de los grupos funcionales asociados a $E$. azurea estuvo representada principalmente por los predadores $(54,81 \%)$, con 15 familias correspondientes a los órdenes Odonata, Hemíptera, ciertas familias de coleópteros (Dytiscidae, Noteridae y Staphylinidae), así como dípteros (Chaoboridae) y Trichopteros (Polycentropodidae). Le siguen los colectores (43,70\%) representados por 4 familias de los órdenes Díptera, Ephemeroptera y coleópteros de la familia Elmidae y finalmente se encuentran los fragmentadores $(1,48 \%)$ con dos familias (Figura 3).

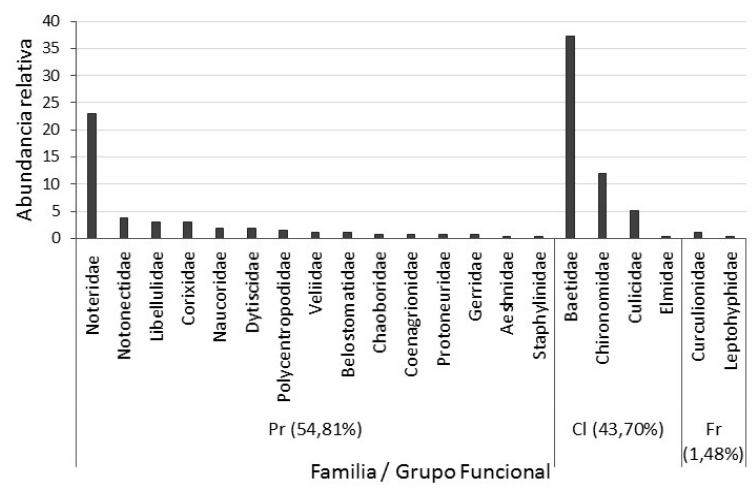

Figura 3. Abundancia relativa de familias y grupos funcionales de los insectos acuáticos asociados a las raíces de $E$. azurea en la zona de estudio. Pr: predador, $\mathrm{Cl}$ : colector, Fr: fragmentador.

Tabla 1. Composición taxonómica de los insectos acuáticos asociados a las raíces de Eichhornia azurea.

\begin{tabular}{|c|c|c|c|c|c|c|}
\hline Clase & Orden & Familia & Género & Aguas altas & Aguas bajas & Grupo funcional \\
\hline \multirow{28}{*}{ Insecta } & \multirow{8}{*}{ Diptera } & \multirow{3}{*}{ Culicidae } & Orthopomyia & 7 & 0 & $\mathrm{Cl}$ \\
\hline & & & Chagasia & 3 & 4 & $\mathrm{Cl}$ \\
\hline & & & Ablabesmyia & 17 & 4 & $\mathrm{Cl}$ \\
\hline & & \multirow{2}{*}{ Chironomidae } & Ortochladius & 1 & 0 & $\mathrm{Cl}$ \\
\hline & & & Pentaneura & 10 & 0 & $\mathrm{Cl}$ \\
\hline & & \multirow[t]{2}{*}{ Chaoboridae } & Chaoborus & 0 & 2 & $\mathrm{Pr}$ \\
\hline & & & Americabaetis & 0 & 1 & $\mathrm{Cl}$ \\
\hline & & \multirow[t]{2}{*}{ Baetidae } & Paracloeodes & 0 & 3 & $\mathrm{Cl}$ \\
\hline & \multirow{4}{*}{ Ephemeroptera } & & Callibaetis & 0 & 96 & $\mathrm{Cl}$ \\
\hline & & Leptohyphidae & Tricorythodes & 0 & 1 & $\mathrm{Fr}$ \\
\hline & & Dytiscidae & Rhantus & 0 & 1 & $\mathrm{Pr}$ \\
\hline & & Dytıscidae & Laccophilus & 3 & 1 & $\mathrm{Pr}$ \\
\hline & \multirow{6}{*}{ Coleoptera } & Elmidae & Hexanchorus & 0 & 1 & $\mathrm{Cl}$ \\
\hline & & Curculionidae & Tanysphyrus & 2 & 1 & Fr \\
\hline & & Staphylinidae & Stenus & $\overline{1}$ & 0 & $\mathrm{Pr}$ \\
\hline & & Noteridae & Hydrocanthus & 59 & 3 & $\mathrm{Pr}$ \\
\hline & & Coenagrionidae & Acanthagrion & 0 & 1 & $\mathrm{Pr}$ \\
\hline & & Coenagrionidae & Ischnura & 0 & 1 & $\mathrm{Pr}$ \\
\hline & \multirow[t]{6}{*}{ Odonata } & Protoneuridae & Protoneura & 0 & 2 & $\operatorname{Pr}$ \\
\hline & & Libellulidae & Tramea & 5 & 3 & $\mathrm{Pr}$ \\
\hline & & Aeshnidae & Boyeria & 0 & 1 & $\mathrm{Pr}$ \\
\hline & & Veliidae & Paravelia & 0 & 3 & $\mathrm{Pr}$ \\
\hline & & Corixidae & Tenagobia & 7 & 1 & $\mathrm{Pr}$ \\
\hline & & Naucoridae & Pelocoris & 1 & 4 & $\operatorname{Pr}$ \\
\hline & \multirow{3}{*}{ Hemiptera } & Gerridae & Trepobates & 2 & 0 & $\mathrm{Pr}$ \\
\hline & & Notonectidae & Notonecta & 0 & 10 & $\mathrm{Pr}$ \\
\hline & & Belostomatidae & Belostoma & 1 & 2 & $\operatorname{Pr}$ \\
\hline & Trichoptera & Polycentropodidae & Cyrnellus & 1 & 3 & $\mathrm{Pr}$ \\
\hline
\end{tabular}

*Grupo funcional: (Pr) Predadores, (Cl) Colectores, (Fr). Fragmentadores. 
Variables físico-químicas y comunidad de insectos acuáticos asociados a $E$. azurea. La Tabla 2 resume los datos estadísticos básicos de las variables abióticas analizadas; con las variables conductividad eléctrica, transparencia y sólidos totales disueltos (STD) como las de mayor variación a lo largo del estudio; sin embargo, ninguna de las variables analizadas presentó diferencias significativas entre periodos hidrológicos $(p>0,05)$.

Tabla 2. Valores promedios, rangos, coeficientes de variación (CV) y diferencia significativa para las variables abióticas entre periodos hidrológicos.

\begin{tabular}{lcccc}
\hline \multicolumn{1}{c}{ Variables } & $\mathbf{P}$ & $\mathbf{R}$ & $\mathbf{C V} \%$ & $\mathbf{P V}$ \\
\hline Oxígeno disuelto $\left(\mathrm{mg} . \mathrm{I}^{-1}\right)$ & 5,2 & $4,26-6,74$ & 17,72 & 0,35 \\
$\mathrm{pH}$ (unidades) & 6,8 & $6,38-7,38$ & 5,03 & 0,16 \\
Temperatura del agua $\left({ }^{\circ} \mathrm{C}\right)$ & 28,3 & $27,3-29,6$ & 3,15 & 0,24 \\
Conductividad eléctrica $(\mu \mathrm{S} / \mathrm{cm})$ & 119,6 & $27,24-431$ & 130,52 & 0,06 \\
Alcalinidad $\left(\mathrm{mg} . \mathrm{I}^{-1} \mathrm{CaCO}\right)$ & 18,2 & $11-25$ & 31,07 & 0,16 \\
STD $\left(\mathrm{mg} . \mathrm{I}^{-1}\right.$ ) & 39,1 & $11,05-78$ & 66,87 & 0,81 \\
Transparencia $(\mathrm{m})$ & 0,7 & $0,25-1,51$ & 72,45 & 0,35 \\
Profundidad $(\mathrm{m})$ & 1,9 & $0,97-3,13$ & 47,95 & 0,64 \\
\hline P=Promedio; R=Rango; PV=p-Valor.
\end{tabular}

La Tabla 3 muestra las correlaciones entre las variables abióticas y la comunidad de insectos acuáticos $(p<$ 0,05 ). A través de la correlación de Pearson se verificó que las variables que más influencian la comunidad de insectos acuáticos asociados a $E$. azurea fueron oxígeno disuelto, $\mathrm{pH}$, conductividad eléctrica, sólidos totales disueltos (STD), transparencia y profundidad. Se resalta a los hemípteros, ya que varios géneros se correlacionan con estas variables; igualmente, la conductividad eléctrica fue la variable que se correlaciono con el mayor número de taxones.

Tabla 3. Correlación de Pearson $(p<0,05)$ entre las variables abióticas y los taxones de insectos acuáticos asociados a $E$. azurea.

\begin{tabular}{|c|c|c|c|c|c|c|}
\hline & OD & $\mathrm{pH}$ & Cond. & Transp. & STD & Prof. \\
\hline $\begin{array}{c}\text { Chaoborus } \\
\text { Chagasia }\end{array}$ & & & 0,98 & 0.92 & & \\
\hline Americabaetis & & & 0,98 & & & \\
\hline Paracloeodes & & & 0,98 & & & \\
\hline Callibaetis & 0,84 & & & & 0,82 & \\
\hline Tricorythodes & & & 0,98 & & & \\
\hline $\begin{array}{c}\text { Rhantus } \\
\text { Hexanchorus }\end{array}$ & $\begin{array}{l}0,84 \\
0,84\end{array}$ & & & & $\begin{array}{l}0,82 \\
0,82\end{array}$ & \\
\hline $\begin{array}{l}\text { Acanthagrion } \\
\text { lschnura }\end{array}$ & $\begin{array}{l}0,84 \\
084\end{array}$ & & & & $\begin{array}{l}0,82 \\
0,82\end{array}$ & \\
\hline $\begin{array}{c}\text { Protoneura } \\
\text { Boyeria }\end{array}$ & & & $\begin{array}{l}0,98 \\
0,98\end{array}$ & & & \\
\hline Tenagobia & & 0,85 & & & & \\
\hline $\begin{array}{l}\text { Paravelia } \\
\text { Pelocoris }\end{array}$ & 0,84 & & 092 & & 0,82 & \\
\hline Belostoma & & & 0,81 & 0,78 & & 0,87 \\
\hline Notonecta & & & 0,98 & & & \\
\hline Trepobates & & 0,84 & & & & \\
\hline
\end{tabular}

$\mathrm{OD}=$ oxígeno disuelto; Cond. = conductividad; Transp. = transparencia; STD = sólidos totales disueltos; Prof. = profundidad

\section{DISCUSIÓN}

Composición y estructura por grupos funcionales de la comunidad de insectos acuáticos asociados a $E$. azurea. Durante este estudio $E$. azurea presentó una rica fauna de insectos acuáticos, demostrando ser un recurso atractivo para esta comunidad; con una composición taxonómica similar a la reportada en otras investigaciones realizadas en el país en macrófitas del mismo género, como E. crassipes (DELUQUE et al., 2006; QUIROS et al., 2010; RIVERA-USME et al., 2013; RÚA-GARCÍA, 2015). Esta riqueza puede darse ya que esta planta acuática flotante, presenta una extensa y ramificada red de raíces, además de que el tallo y gran parte de sus hojas están en contacto con la columna de agua, presentando mayor diversidad de microhábitats para las comunidades acuáticas (SCHEFFER, 2004; TOWNSEND et al., 2010). Así mismo, de acuerdo con THOMAZ y CUNHA (2010) y QUIROZ et al. (2008), este tipo de vegetación proporciona alimento, refugio, áreas para colonización y reproducción, promoviendo un incremento en la biodiversidad, así como en las relaciones inter e intraespecíficas.

La mayor abundancia de organismos y de taxones se registró en aguas bajas. De acuerdo con TUNDISI y MATSUMURA-TUNDISI (2008) y SYCHRA et al. (2010), los cambios en profundidad ejercen influencia en la distribución de la macrofauna entre las raíces de las macrófitas, contribuyendo de esta forma a diferentes distribuciones de abundancia y riqueza, como las observadas en la presente investigación.

La alta abundancia del orden Ephemeroptera, particularmente Baetidos del género Callibaetis se encuentra quizás relacionada con la capacidad que éstos tienen de utilizar eficientemente los detritus y las algas como fuente de energía (BERNER y PESCADOR, 1988), los cuales son recursos abundantes en las macrófitas acuáticas, como resultado de los procesos de fragmentación de las hojas y liberación de nutrientes. De acuerdo con FLOWERS y DE LA ROSA (2010), este género es común en charcos y lagos, sobre todo con abundante vegetación acuática, tolerando amplios rangos de temperatura, oxígeno disuelto y $\mathrm{pH}$ (ZÚÑIIGA et al., 2004).

Los órdenes Coleoptera, Diptera y Hemiptera son los de mayor riqueza durante el presente estudio; riqueza que se encuentra asociada a la capacidad que estos tienen de adaptarse a múltiples ambientes y desarrollar diferentes estrategias de alimentación, lo que los convierte en grupos importantes en las cadenas tróficas como fuente de alimento para peces y anfibios (ARCHANGELSKY, 2001; MOSQUERA et al., 2008; MAZZUCCONI et al., 2009); todo esto unido a la tolerancia a las altas temperaturas, bajas concentraciones de oxígeno y altas 
concentraciones de materia orgánica (RÚA-GARCÍA, 2015). Particularmente los dípteros son conocidos por presentar un alto número de especies en ambientes lénticos (HIGUTI y TAKEDA, 2002; HIGUTI, 2004), al utilizar a las macrófitas acuáticos como sustrato, gracias a su capacidad de ocupar un amplio rango de microhábitats; a su vez algunos tienen la capacidad de tolerar pH ácidos y bajos valores de oxígeno (PINDER, 1986; PAGGI, 2009).

Los ecosistemas lénticos tienen habitualmente baja diversidad y riqueza, lo cual está relacionado con la poca disponibilidad de hábitats, puesto que las macrófitas y el sedimento del fondo constituyen los únicos sustratos disponibles; lo cual explica los bajos resultados que se registran en este estudio en cuanto a diversidad. De acuerdo con THOMAZ y CUNHA (2010), el incremento en la diversidad y la composición de comunidades de diferentes grupos de organismos adheridos a la vegetación flotante está fuertemente influenciada por la complejidad de macrófitas en diferentes escalas espaciales; con factores como la morfología y textura de macrófitos, el sustrato para la colonización perifítica y la capacidad de retención de materia orgánica en partículas, los que más se relacionan con la riqueza de taxones presentes (POI DE NEIFF y NEIFF, 2006).

Los grupos funcionales registrados en este estudio coinciden con los reportados en otros trabajos realizados en $E$. azurea como los de POI DE NEIFF (2003) y SAULINO y TRIVINHO-STRIXINO (2014), quienes reportan a los colectores y predadores como los grupos dominantes. Las extensas raíces fasciculadas de $E$. azurea pueden promover una gran retención de materia orgánica en partículas y la acumulación de detritos, lo que según TRIVINHO-STRIXINO et al. (2000) y MORMUL et al. (2006) favorece la presencia de organismos colectores, los cuales son recursos alimentarios para los depredadores, quienes se encontraron en abundancia en este estudio.

Variables fisicoquímicas y comunidad de insectos acuáticos asociados a $E$. azurea. Los parámetros fisicoquímicos presentaron un comportamiento enmarcado dentro de lo que corresponde a la zona tropical, con valores promedio de oxígeno disuelto inferiores a 5,0 mg. $\mathrm{I}^{-1}$, $\mathrm{pH}$ ligeramente ácido y alta temperatura. De acuerdo con LEWIS (2000) y ROLDAN (2003) es común que en los sistemas lénticos tropicales se presente cierto déficit de oxígeno. En el caso del $\mathrm{pH}$, sus valores son atribuibles a la naturaleza de los suelos de la zona, los cuales son ácidos; sin embargo se encuentran dentro los límites para la supervivencia de los organismos acuáticos (4,5 a 8,5, ROLDÁN y RAMÍREZ 2008). La poca variación observada para la temperatura es una característica de los ecosistemas tropicales, donde las temperaturas no sufren grandes variaciones a lo largo del año, como las que ocurren en las zonas templadas debido a los cambios estacionales (ROLDAN et al., 2001).

La alcalinidad, la conductividad eléctrica y los sólidos totales disueltos se encontraron dentro de los rangos reportados para ecosistemas neotropicales, con valores

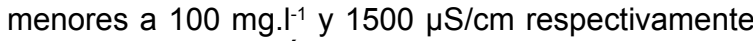
(ROLDAN y RAMÍREZ, 2008). En cuanto a la transparencia, su alta variación se encuentra asociada a la baja profundidad de las ciénagas estudiadas, estando dentro del rango establecido para las ciénagas en Colombia por ARIAS (1985) de entre 0,17 y 1,13 m.

Con respecto a las relaciones arrojadas con los insectos acuáticos, un gran número de taxones mostró correlación con los parámetros ambientales. SOUZA et al. (2009) relaciona las variables ambientales con los insectos acuáticos asociados a $E$. azurea en un lago de inundación del río Paraná (Brasil), encontrando que la temperatura, oxigeno, turbiedad y nutrientes son las variables que más influencian la comunidad de insectos acuáticos. Según autores como COVICH et al. (1999), KREBS (2000) y GRILLET et al. (2002), la estructura y distribución espacial de los macroinvertebrados en las ciénagas se haya asociada a las características físicas, al estado trófico, a la heterogeneidad de hábitats, la profundidad, el oxígeno, el $\mathrm{pH}$, la temperatura y la materia orgánica entre otros factores. Así mismo, de acuerdo con OCON y RODRÍGUEZ (2004) y DOMÍNGUEZ y FERNÁNDEZ (2009), los factores fisicoquímicos son considerados como los aspectos que más influencia ejercen sobre la distribución, abundancia y riqueza de insectos acuáticos.

Los resultados de la presente investigación evidencian la capacidad potencial de las macrófitas acuáticas para albergar una considerable riqueza y abundancia de insectos acuáticos, proporcionando condiciones para la supervivencia y reproducción de estos organismos; lo que posibilita el desarrollo de una fauna diversificada. Así mismo, las correlaciones halladas, aunque preliminares, podrán utilizarse en futuros trabajos que intenten modelar los efectos de los factores ambientales sobre los macroinvertebrados en humedales similares de la región. Por otro lado, el estudio de los grupos funcionales permite tener una visión del estado ecológico de las ciénagas, ya que estos gremios reflejan los atributos relacionados con los ensamblajes de la comunidad desde una perspectiva funcional.

\section{Agradecimientos.}

A Grupo de Zoología, líneaictiología de la Universidad Tecnológica del Chocó por el financiamiento de la presente investigación. Al laboratorio de Limnología delaUniversidad Tecnológica del Chocó porla logística delaboratorio.Alos estudiantes JoeA. Murillo, Wilber Pino por su apoyo en la fase de campo. 


\section{REFERENCIAS}

Álvarez, J.C.; Vivas, I. 1999. Variación espacial de la estructura de macroinvertebrados asociados a raíces de Pontederia rotundifolia (Algamasa) de la zona litoral y limnética en las ciénagas Plaza Seca y La Grande, Sanceno, Quibdó - Colombia. Trabajo de grado. Facultad de Ciencias Básicas. Universidad Tecnológica del Chocó, Quibdó, Chocó.

Álvarez, J.; Cuesta, Y.; Asprilla, S. 2003. Estudio comparativo de bentos y organismos asociados a macrófitas en un ambiente acuático temporal, Quibdó-Chocó. Revista Institucional Universidad Tecnológica del Chocó 19:64-69.

ARCHANGELSKY, M. 2001. Coleóptera. En: Fernández y Domínguez (Eds). Guía para la determinación de artrópodos bentónicos sudamericanos. Editorial Universitaria de Tucumán, Argentina.

Arias, P.A. 1985. Las ciénagas en Colombia. Divulgación Pesquera 22 (3-5):38-70.

Asprilla, S.; Ramírez, J.J; Roldán, G. 1998. Caracterización Limnológica Preliminar de la Ciénaga de Jotaudó (Chocó, Colombia). Revista Actualidades Biológicas 20 (69):87-107.

Berner, L.; Pescador, M.L. 1988. The mayflies of Florida, Revised edition. Tallahassee/Gainesville: University Presses of Florida.

CHARÁ-SERNA, A.M.; CHARÁ, J.D.; ZÚÑIGA, M. DEL C.; PEDRAZA, G.X.; GIRALDO, L.P. 2010. Clasificación trófica de insectos acuáticos en ocho quebradas protegidas de la ecorregión cafetera colombiana. Universitas Scientiarum 15 (1):27-36. https://doi.org/10.11144/javeriana.SC15-1.tcoa

COVICH, A.; PALMER, M.; CROWL, T. 1999. Macroinvertebrates role in water ecosystem. Bioscience 49 (2):119127. https://doi.org/10.2307/1313537

Deluque, J.; Reyes, S.; Sierra-Labastidas, T.; Lopez, W. 2006. Primeros reportes de familias de macroinvertebrados asociados a macrófitas acuáticas en la ciénaga del Cerro de San Antonio (río Magdalena, Colombia. Revista Intrópica 3:77-86.

Domínguez, E.; Fernández, H.R. (Ed.). 2009. Macroinvertebrados Bentónicos Sudamericanos. Fundación Miguel Lillo, Tucumán, Argentina. .

Domínguez, E.; Molineri, C.; Pescador, M.I.; Hubbard, M.D.; Nieto, C. 2006. Ephemeroptera of South América. In: Adis, J., Arias J.R., Rueda-Delgado, G., Wantzen, K.M. (Eds): Aquatic Biodiversity en Latin América (ABLA), SofiaMoscow Pensoft, 2.

Duque, H. 1990. Estratigrafía, Paleoceanografía y Paleobiogeografía de la Cuenca del Atrato y la Evolución del Istmo de Panamá. Ingeominas. Bogotá.

Flowers, R.W.; De La Rosa, C. 2010. Ephemeroptera. Ver. Biol. Trop. 58 (Suppl. 4):63-93.

FONTANARROSA, M.S.; CHAPARRO, G.N.; O'FARRELL, I. 2012. Temporal and spatial patterns of macroinvertebrates associated with small and medium-sized free-floating plants. Wetlands 33:47-63. https://doi.org/10.1007/s13157012-0351-3

Grillet, M.; Legendre, P.; Borcard, D. 2002. Community structure of Neotropical wetland insects in Northern Venezuela: I. Temporal and environmental factors. Arch. Hydrobiol 155:413-436. https://doi.org/10.1127/archivhydrobiol/155/2002/413

HAMMER, O.; HARPER, D.; RYAN, P.D. 2001. Past: Paleontological Statistics Software Package for Education and Data Analysis. Palaeontologia Electronica 4. Disponible en: http://palaeoelectronica. Org /2001_1/past/issue1_01.htm.

Higuti, J. 2004. Spatial and temporal variation in densities of chironomid larvae (Diptera) in two lagoons and two tributaries of the upper Paraná River floodplain, Brazil. Brazilian Journal of Biology 62 (4):807-818. 
Higuti, J.; Takeda, A.M. 2002. Composition, density and habitats of benthic chironomid larvae. Págs. 209-221.En: Thomaz, S.M.; Agostinho, A.A.; Hahn, N.S. (Ed.). The upper Paraná River and its floodplain: physical aspects, ecology and conservation. Leiden: Backhuys Publishers.

Inger, D.J.; Deluque, S.; Reyes-Sierra, T. 2004. Composición de la Comunidad de Macroinvertebrados Acuáticos Asociados a las Macrófitas de la Ciénaga del Cerro San Antonio. VI Seminario Colombiano de Limnología, Montería, Colombia:49.

INSTITUTO GEOGRÁFICO AGUSTÍN CODAZZI «IGAC». 2006. Chocó: Características Geográficas. BogotáColombia.

Krebs, C.H. 2000. Ecología: estudio de la distribución y la abundancia. 2da Edición en espa-ol. Oxford University Press. México.

LEWIS, M. 2000. Basis for the protection and management of tropical lakes. Lakes and Reservoirs: Research and Management 5:35-48. https://doi.org/10.1046/j.1440-1770.2000.00091.x

MANJARRÉS-HERNÁNDEZ, A.; FLÓREZ-LEIVA, A.L; RUEDA-DELGADO, G. 2004. Macrófitas acuáticas de la ciénaga de San Antonio - Magdalena, Colombia. Págs. 75-77. En: ACL - Limnos (Ed). Resúmenes VI Seminario Nacional de Limnología y I Encuentro Internacional sobre Embalses Neotropicales. Neolimnos 2004. Universidad Pontificia Bolivariana-Asociación Colombiana de Limnología. Montería, Colombia.

Martínez, M.A. 2009. Macroinvertebrados acuáticos como indicadores de calidad del agua en tres ciénagas del departamento de Cesar, Colombia. Tesis de maestría. Facultad de Ciencias, Universidad Nacional de Colombia, Bogotá.

MARTINS, R.T.; SILVEIRA, L. S.; ALVES, R. G. 2011. Colonization by oligochaetes (Annelida: Citellata) in decomposing leaves of Eichhornia azurea (SW.) Kunth (Pontederiaceae) in a tropical lentic system. Annales de Limnologie International Journal of Lymnologi 47:339-346. https://doi.org/10.1051/limn/2011053

Mazzucconi, S.; López-Ruf, M.L.; Backmann, A.O. 2009. Hemiptera-Heteroptera: Gerromorpha y Nepomorpha. En: Domínguez, E., Fernández, H.R. (Eds.) Macroinvertebrados bentónicos sudamericanos: Sistemática y biología. Fundación Miguel Lillo. Tucumán, Argentina.

MERRITT, R.W.; CUMMINS, K.W.; BERG, M.B. 2008. An introduction to the aquatic insects of North America. Dubuque, Kendall/Hunt Publishing Company.

MINITAB INC 17.1.0. 2014. Minitab Statistical Software. State College, Pennsylvania: Minitab Inc.

Moretti, M.S.; Goulart, M.D.C.; Callisto, M. 2003. Avaliação rápida da macrofauna associada à Eichhornia azurea (Swartz) Kunth, 1843 e Pontederia laceolata Nutt., 1818 (Pontederiaceae) na Baía do Coqueiro, Pantanal de Poconé (MT/Brasil). Revista Brasileira de Zoociencias 5:7-21.

Mormul, R. P.; Vieira, L. A.; Pressinatte jr, S.; Monkolski, A.; Santos, A. M. 2006. Sucessão de invertebrados durante o processo de decomposição de duas plantas aquáticas (Eichhornia azurea e Polygonum ferrugineum). Acta Scientiarum. Biological Sciences 28 (2):109-115.

Mosquera, D.R.; Palacios, M.L.; Muñoz, E.; Soto, A.; Peña, E.J. 2008. Diversidad de los macroinvertebrados de la laguna de Sonso, Valle del Cauca, Colombia. Revista de Ciencias 12:45-56.

Mosquera-Murillo, Z; Córdoba-Aragón, K. 2015. Caracterización de la comunidad de macroinvertebrados acuáticos en tres ciénagas de la cuenca media del Atrato, Chocó - Colombia. Revista Institucional Universidad Tecnológica del Chocó 34 (1):22-35.

OCON, C.S.; RODRÍGUEZ, A. 2004. Presence and abundance of Ephemeroptera and other sensitive macroinvertebrates in relation with habitat conditions in Pampean streams (Buenos Aires, Argentina). Arch. Hydrobiol 159:473-487. https://doi.org/10.1127/0003-9136/2004/0159-0473 
OVALLE, H. 2006. Distribución espacial y temporal de la comunidad de macroinvertebrados acuáticos del humedal el Jaboque (Bogotá, Colombia). Serial online. Acceso 22 de agosto 2010. Disponible en http://www.ramsar.org/pdf/ wwd/6/wwd2006_rpts_colombia01.pdf.

PADIAL, A.A.; THOMAZ, S.M.; AGOSTINHO, A.A. 2009. Effects of structural heterogeneity provided by the floating macrophytes Eichhornia azurea on the predation efficiency and habitat use of small Neotropical fish Moenkhausia sanctaefilomenae. Hydrobilogia 624:161-170. https://doi.org/10.1007/s10750-008-9690-8

Paggi, A.C. 2009. Diptera. In: E. Domínguez \& H.R. Fernández (Eds.), Macroinvertebrados Bentónicos Sudamericanos: Sistemática Y Biología (pp. 383-410). Tucumán - Argentina: Fundación Miguel Lillo.

PINDER, L. 1986. Biology of Freshwater Chironomidae. Annual Review of Entomology 31(1):1-23. https://doi. org/10.1146/annurev.en.31.010186.000245

Poi De Neiff, A. 2003. Macroinvertebrates living on Eichhornia azurea Kunth in the Paraguay River. Acta Limnol. Bras 15:55-63.

Poi De Neiff, A.; Neiff, J.J. 2006. Riqueza de especies y similaridad de los invertebrados que viven en plantas flotantes de la Planicie de Inundación del Río Paraná. Interciência 31(3):220-225.

Pott, V.J.; Pott, A. 2000. Plantas aquáticas do Pantanal. Brasília: Embrapa - Centro de Pesquisa Agropecuária do Pantanal.

Quirós-Flores, A.; Miranda-Arce, M.G.; Lot-Helgueras, A. 2008. Estudio comparativo de algunas variables fisicoquímicas del agua en canales secundarios de Xochimilco con y sin Eichhornia crassipes (Martius) SolmsLaubach. Polibotanica 25:127-133.

Quirós-Rodríguez, J.A.; Dueñas Ramírez, P.R.; Ballesteros Correa, J. 2010. Macroinvertebrados asociados a las raíces de Eichhornia crassipes (Mart). Solms, en dos sectores del complejo cenagoso del bajo Sinú, departamento de córdoba, Colombia. Rev. Asoc. Col. Cienc. (Col.) 22:147-157.

RANGEL, J.O. 2004. Colombia Diversidad Biótica IV: El Chocó Biogeográfica Costa Pacífica. Universidad Nacional de Colombia. Bogotá. Ed. UNAL. 996 p.

Ríos, L.; Palacios, J.; Aguirre, N. 2008. Variabilidad fisicoquímica del agua en la ciénaga El Eneal, reserva natural Sanguaré municipio de San Onofre-Sucre, Colombia. Rev Fac Ing Univ Antioquia 46 (1):39-45.

Rivera-Usme, J.J.; Pinilla-Agudelo, G.; Camacho-Pinzón, D.L. 2013. Grupos tróficos de macroinvertebrados acuáticos en un humedal urbano andino de Colombia. Acta biol. Colomb. 18 (2):279-292.

Rivera-Usme, J.J. 2011. Relación entre la composición y biomasa de la comunidad de macroinvertebrados acuáticos y las variables físicas y químicas en el humedal Jaboque, Bogotá-Colombia. Tesis de Maestría. Universidad Nacional de Colombia, Bogotá, Colombia.

Rivera-Usme, J.J.; Pinilla Agudelo, G.; Camacho Pinzón, D.L. 2013. Grupos tróficos de macroinvertebrados acuáticos en un humedal urbano andino de Colombia. Acta biol. Colomb. 18 (2):279-292.

Roldán, G. 2003. Bioindicación de la calidad del agua en Colombia. Uso del método BMWP/Col. Universidad de Antioquia, Medellín, Antioquia, Colombia.

Roldán, G.; Bohórquez, A.; Cataño, R.; Ardila, J. 2001. Estudio Limnológico del Embalse del Guavio. Revista de la Academia Colombiana de Ciencias Exactas Físicas y Naturales 24 (90):25-33.

Roldán, G.; Ramírez, J.J. 2008. Fundamentos de Limnología Neotropical. $2^{a}$ ed. Universidad de Antioquia-ACCEFYNUniversidad Católica de Oriente, Medellín. 
RÚA-GARCÍA, G. 2015. Macroinvertebrados acuáticos asociados a raíces de Eichhornia crassipes (Mart) Solms, en la ciénaga de Zapayán, Magdalena-Colombia. Rev. Intropica 10:52-59. https://doi.org/10.21676/23897864.1647

SAULINO, H.E.; TRIVINHO-STRIXINO, S. 2014. Macroinvertebrados aquáticos associados às raízes de Eichhornia azurea (Swarts) Kunth (Pontederiaceae) em uma lagoa marginal no Pantanal, MS. Biotemas 27 (3):65-72. https:// doi.org/10.5007/2175-7925.2014v27n3p65

Scheffer, M. 2004. Ecology of shallow lakes. Norwell: Kluwer Academic Publishers, 357 p. https://doi.org/10.1007/9781-4020-3154-0

Souza-Franco, G.M.; Andrian, L.F.; Franco, R.M. 2009. Comunidade de insetos aquáticos associados à Eichhornia azurea (Schwartz) Kunth, em uma lagoa de várzea na planície de inundação do Alto Rio Paraná, Mato Grosso do Sul, MS, Brasil. Biológico 71 (1):83-91.

STATGRAPHICS CENTURION XV. 2006. [programa de ordenador]. Versión Centurion XV. E.U.A: Stat Point, Inc.

STRIPARI, N.L.; HENRY, R. 2002. The invertebrate colonization during ecomposition of Eichhornia azurea Kunth in a lateral lake in the mouth zone of Paranapanema River into Jurumirim Reservoir (São Paulo, Brazil). Brazilian Journal of Biology 62 (2):293-310. https://doi.org/10.1590/S1519-69842002000200014

SYCHRA, J.; ADAMÉK, Z.; PETIVALSKÁ. 2010. Distribution and diversity of littoral macroinvertebrates within extensive reed beds of lowland pond. Annals de Limnologie - International Journal of Lymnologi 46:281-289. https:// doi.org/10.1051/limn/2010026

THOMAZ, S.M.; CUNHA, E.R.D. 2010. The role of macrophytes in habitat structuring in aquatic ecosystems: methods of measurement, causes and consequences on animal assembleges's composition and biodiversity. Acta Limnologica Brasiliensia 22 (2):218-236. https://doi.org/10.4322/actalb.02202011

Townsend, C.R.; Begon, M.; Harper, J.L. 2010. Fundamentos em ecologia. Porto Alegre: Artmed.

TRIVINHO-STRIXINO, S.; CORREIA, L.S.C.; SONODA, K. 2000. Phytophilous Chironomidae (Diptera) and other macroinvertebrates in the ox-bow Infernão Lake (Jataí Ecological Station, Luiz Antônio, SP, Brazil). Revista Brasileira de Biologia 60 (3):527-535. https://doi.org/10.1590/S0034-71082000000300018

TRIVINHO-STRIXINO, S.; RODRIGUES-PELAEZ, M.; JANCSO, M.A. 2007. Chironomidae associados a Eichhornia azurea (Swarts) Khunt: influência da profundidade do corpo drágua na estrutura da comunidade fitófila de lagoas marginais do Rio Mogi-Guaçu (Luiz Antonio, SP). Págs. 26-33. En: SIMPÓSIO DE ECOLOGIA do PPGERN/UFSCar, I, São Carlos. Anais Cadernos de Resumos expandidos, São Carlos: PPGERN/UFSCa.

Tundisi, J.G.; Matsumura-Tundisi, T. 2008. Limnologia. São Paulo: Oficina de Textos. Brasil.

Venegas, E.M. 2008. Estado limnológico de cuatro humedales de la sabana de Bogotá utilizando macroinvertebrados como bioindicadores. Trabajo de Grado. Departamento de Biología, Facultad de Ciencias, Universidad Nacional de Colombia.

VILLANUEVA, V.D.; TROCHINE, C. 2005. The role of microorganisms in the diet of verger CF. Limnophilus (Trichoptera: Limnephilidae) larvae in the Patagonian Andean temporary pond. Wetlands 25 (2):473-479. https:// doi.org/10.1672/21.1

WALKER, P.D.; WIJNHOVEN, S.; VELDE, G.V.D. 2013. Macrophyte presence and growth form influence macroinvertebrate community structure. Aquatic Botany 104:80-87. https://doi.org/10.1016/j.aquabot.2012.09.003

Zúñiga, M.C.; Molineri, C.; Dominguez, E. 2004. El orden Ephemeroptera en Colombia. In: Fernandez, F.C., Andrade-C, M.G. y Amat, G.D. (Eds.). Insectos de Colombia, 